\title{
Preparation and Characterization
}

\section{of Surfactant-Modified Powder Activated Carbon (SM-PAC) Reinforced Poly (Ethylene Oxide) (PEO) Composites}

\author{
A. GÜRSES ${ }^{a}$, M. Ejder-Korucu ${ }^{b, *}$ And Ç. DoĞAR ${ }^{c}$ \\ ${ }^{a}$ Atatürk University, Faculty of K.K. Education, 25240, Erzurum, Turkey \\ ${ }^{b}$ Kafkas University, Faculty of Science and Literature, 361000, Kars, Turkey \\ ${ }^{c}$ Erzincan University, Faculty of Education, 24030, Erzincan, Turkey
}

\begin{abstract}
Poly (ethylene oxide), PEO, which was used for a wide variety of applications is a flexible, crystalline, thermoplastic, water-soluble and non-ionic polymer. The most of studies on surfactant-modified activated carbon (SM-PAC) have been generally focused on the removal of contaminants. This study aims the preparation of $\mathrm{PEO} /(\mathrm{SM}-\mathrm{PAC})$ composites, using solution-intercalation method. Firstly activated carbon was modified with cationic surfactant, Cetyltrimethylammonium Bromide (CTAB). Then PEO/(SM-PAC) composites were prepared using solution-intercalation method, with two different (SM-PAC) contents (1.0 and 2.0 wt.\%). The characterization of the composites was made by X-ray diffraction (XRD), Fourier Infrared Spectroscopy (FT-IR), thermal analysis and tensile tests. The XRD patterns revealed that $2 \theta$ positions of the broad peaks belonging to the composites significantly shift to left compared with those of virgin PEO. The SEM images of the surfactant-modified powdered activated carbon (SM-PAC)/PEO composites prepared with the two different SM-PAC contents show that there is an intensive interaction between the $\mathrm{CTA}^{+}$ions of modified activated carbon surface and the polymer chains. From the tensile tests, it was found that the unmodified powdered activated carbon dispersed into the polymer matrix made the ductile polymer more brittle. However, the tensile and yield strengths of the composite, prepared with modified powdered activated carbon, have decreased, and the strain percent value has significantly increased.
\end{abstract}

DOI: 10.12693/APhysPolA.129.849

PACS/topics: 81.70.Pg

\section{Introduction}

The modification of surface characteristics of solid adsorbents such as powder activated carbon (PAC), using surfactants exhibits a practical importance in terms of industrial and environmental applications [1]. Activated carbon is an amorphous, highly porous carbon material with a high specific surface area [2]. The activated carbon with large surface area, high adsorption capacity, and micro porous structure is one of the most effective adsorbents for organic compounds [3-6], and thus plays an important role in modern since and technology. For instance it is used in the purification of liquids and gases $[7,8]$, in the catalysis $[9,10]$, for the separation of mixtures [11, 12], and even in the hydrogen storage $[13,14]$. At a cationic surfactant/activated carbon/water system, the surfactant ion or molecules can adsorb onto the surface of the activated carbon through hydrophobic interactions, and hence their hydrophilic parts are oriented toward the aqueous phase [15-20]. Poly (ethylene oxide) (PEO) is the most commercially important polyether. PEO is polymer of ethylene oxide commercially available in the wide range of molecular weights [21]. PEO has $-\mathrm{CH}_{3}$ end group. $\mathrm{PEO}$ is semi-crystalline, biocompatible, biodegradable, non-ionic

*corresponding author; e-mail: mehtapejderk@gmail.com and water-soluble polymer of considerable industrial significance which finds applications in many different branches of industry [22]. PEO is currently also used in the pharmaceutical industry for applications such as controlled-release, solid-dose matrix systems, transdermal drug delivery systems and mucosal bioadhesives [23]. PEO is also a very suitable material for hot melt extrusion due to its very good processing ability in different processing conditions, where polymer of low molecular weight can act as a plasticizer for high molecular weight polymer [24]. (PEO)/clay nanocomposites offer new promising materials which display a great potential for use in various application fields [25-26]. The hydrophilic nature and very strong polarity of PEO presents an important advantage when preparing intercalates of $\mathrm{PEO}$ and hydrophilic silicate clay platelets [27, 28]. So far, the most of the present studies involving surfactantmodified activated carbon (SM-PAC) have been generally focused on the removal of contaminants. The present study, is aimed on the preparation of $\mathrm{PEO} /(\mathrm{SM}-\mathrm{PAC})$ composites, using solution-intercalation method. Firstly powdered activated carbon was modified with cationic surfactant, Cetyltrimethylammonium Bromide (CTAB). Then $\mathrm{PEO} /(\mathrm{SM}-\mathrm{PAC})$ composites were prepared using solution-intercalation method, with two different (SM-PAC) contents (1.0 and $2.0 \mathrm{wt} . \%)$. The characterization of the composites was made using X-ray diffraction (XRD), Fourier infrared spectroscopy (FT-IR), thermal analysis and tensile tests. 


\section{Materials and methods}

\subsection{Materials}

In this study, Cetyltrimethylammonium Bromide (CTAB) (surfactant or modifier agent) and powdered activated carbon (PAC) (reinforcement filler) were used to produce the polyethylene oxide (PEO)composites. The polyethylene oxide (PEO) with molecular weight of $900.000 \mathrm{~g} / \mathrm{mol}$ was purchased from Aldrich. The Cetyltrimethylammonium Bromide (CTAB) and dichloromethane (Methylene chloride) were purchased from Merck, and were used without further purification.

\subsection{Preparation of surfactant modified activated carbon}

Powder activated carbon was modified by adsorption of Cethyltrimethylammonium Bromide (CTAB) at a concentration above critical micelle concentration (CMC) $(1.0 \mathrm{~g} / \mathrm{g}$ powdered activated carbon). For this, $5.0 \mathrm{~g}$ of PAC were added to the aqueous solution of $1.0 \mathrm{l}$, including $5.0 \mathrm{~g}$ of CTAB. The mixture was placed in a temperature controlled orbital shaker at $160 \mathrm{rpm}$ and $25^{\circ} \mathrm{C}$ for $360 \mathrm{~min}$ and left to attain the ambient conditions. Sample was filtered and dried in an oven overnight at $110^{\circ} \mathrm{C}$.

\subsection{Synthesis of composites}

Polyethylene oxide/surfactant modified powder activated carbon (PEO/SM-PAC) composites were produced by the solution interaction method. The SMPAC was suspended in Methylene chloride for $10 \mathrm{~h}$ in a three-necked flask at ambient temperature and then mixed for $15 \mathrm{~min}$. The $\mathrm{PEO} /$ Methylene chloride ratio, $1: 33(\mathrm{~g} / \mathrm{ml})$, was maintained by adding an additional quantity of solvent as well as PEO. The flask equipped with a condenser, a mechanical stirrer and a thermometer and its content were then placed in an oil bath heated to $80^{\circ} \mathrm{C}$. The flask was kept under stirring condition for $10 \mathrm{~h}$ and then the composites were put into Petri dishes and dried in air overnight at ambient temperature. The same procedure was applied to different SM-PAC ratios (1.0 and 2.0 wt.\%).

\subsection{Characterization of composites}

The composites were analyzed using a Rigaku powder X-ray diffractometer with a $\mathrm{Cu} \mathrm{K}_{\alpha}$ X-ray source $(\lambda=1.5406 \AA)$, and performed at 2 to $40^{\circ}$. The scanning rate of the instrument was $2^{\circ} / \mathrm{min}$. FT-IR Spectra of composites were taken on a Perkin-Elmer SpectrumOne by KBr pelletization method from 400 to $4000 \mathrm{~cm}^{-1}$. Examination of the samples was done with Scanning Electron Microscope (SEM, 400F model Field Emission SEM). Samples were fixed on SEM stubs with a double sided carbon tape and coated with 5-20 nm thick layer of gold/palladium by sputtering. The thermal stability was tested by thermogravimetric analysis (TGA) using Perkin Elmer TGA-7 at a scan rate of $20^{\circ} \mathrm{C} / \mathrm{min}$ in nitrogen.

\section{Results and discussion}

\subsection{XRD and SEM analyses}

$\mathrm{X}$-ray diffraction (XRD) is one of the most important techniques used for the characterization of composites. Figure 1 shows the XRD patterns belonging to PEO, SM-PAC and SM-PAC/PEO (1.0 and 2.0 wt.\%) composites. From this figure, it can be seen that $2 \theta$ positions of the broad peaks belonging to the composites have been significantly shifted to the left compared with those of pure PEO. These shifts clearly indicate the textural and conformational change associated with the rearrangement of PEO chains in the case of composite. In the cases of the composites, the characteristic peaks related with $\mathrm{CTA}^{+}$ions at the modified powdered activated carbon (SM-PAC) have also disappeared. This may imply that there are intensive interactions between the adsorbed $\mathrm{CTA}^{+}$ions and PEO chains.

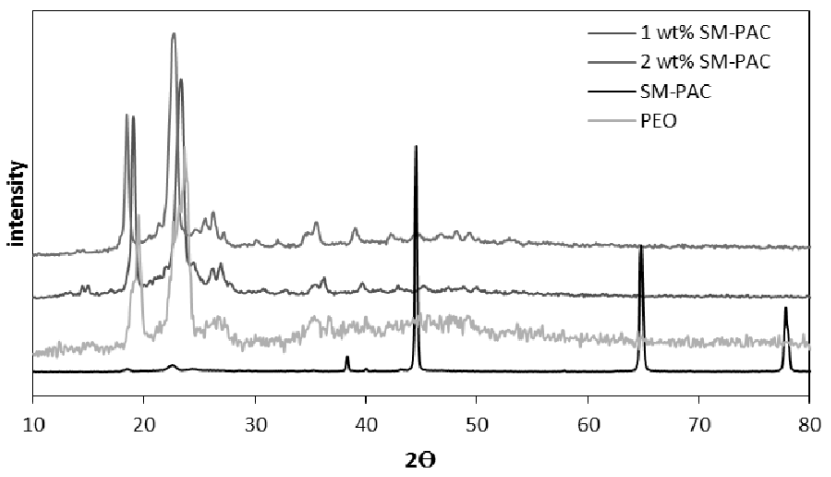

Fig. 1. XRD patterns of pure PEO, SM-PAC and $\mathrm{PEO} / \mathrm{SM}-\mathrm{PAC}$ composites.

SEM images of the samples coded as PAC/CTAB, 1 wt.\% PAC/PEO, 1 wt.\% SM-PAC/ PEO and 2 wt.\% SM-PAC/PEO are given in Fig. 2.

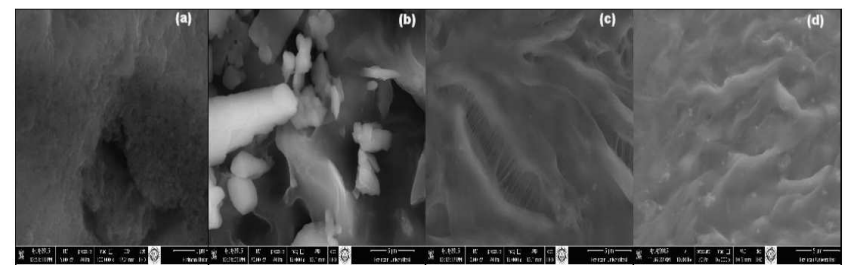

Fig. 2. SEM images of the samples coded as SMPAC (a), 1.0 wt. $\%$ PAC $/ \mathrm{PEO}$ (b), 1.0 wt.\% SM$\mathrm{PAC} / \mathrm{PEO}$ (c) and 2.0 wt.\% SM-PAC/PEO (d).

The SEM image of the surfactant-modified powdered activated carbon (SM-PAC) demonstrates that there is an effective interaction between $\mathrm{CTA}^{+}$ions and the activated carbon surface, which has predominantly occurred via the hydrophobic binding. The SEM image of unmodified powdered activated carbon (PAC)/PEO also shows that the adhesive interactions between polyethylene oxide chains and carbon surface are not clearly effective. 
The SEM images of the surfactant-modified powdered activated carbon (SM-PAC)/PEO composites prepared in the two different SM-PAC contents, 1 and 2 wt.\% (Fig. 2c and $2 \mathrm{~d}$ ), reveal that there is an intensive interaction between the $\mathrm{CTA}^{+}$ions modified activated carbon surface and the polymer chains. Therefore, it can be said that the micelles consisting of $\mathrm{CTA}^{+}$ions have caused a significant change in the arrangement of polymer chains as well as a marked reduction in the polymer/active carbon interfacial energy.

\subsection{FTIR analysis}

FTIR spectra of pure PEO, PAC/PEO, 1.0 wt.\% SM$\mathrm{PAC} / \mathrm{PEO}$ and 2.0 wt.\% SM-PAC/PEO are given in Fig. 3.

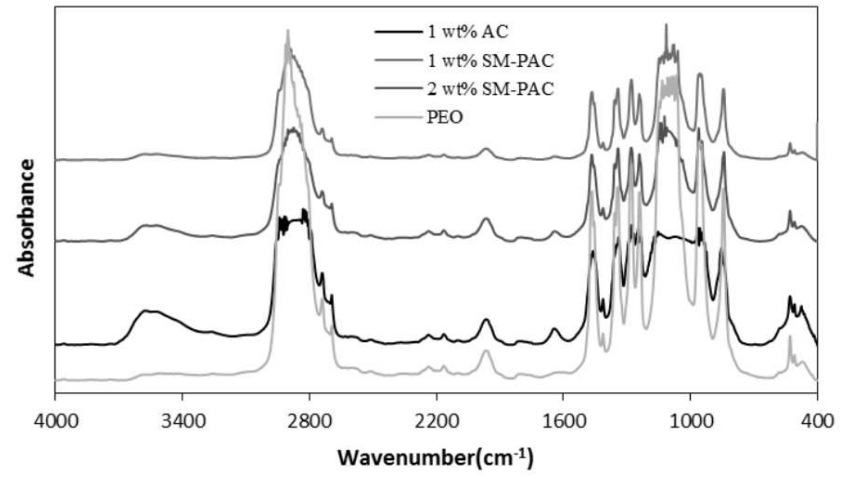

Fig. 3. FTIR spectra of pure PEO, PAC/PEO, 1.0 wt.\% SM-PAC/PEO and 2.0 wt.\% SM-PAC/PEO.

PEO has a helical structure in the crystalline state. The conformation along this chain is nearly trans (CC$\mathrm{CO})$, trans (CO-CC) and gauche (OC-CO). The $\mathrm{CH}_{2}$ rocking modes found in the $1000-700 \mathrm{~cm}^{-1}$ region are particularly sensitive to conformational changes [27]. Pure PEO exhibits a broad band of asymmetric $\mathrm{CH}_{2}$ stretching between 2945-2891 $\mathrm{cm}^{-1}$ and two narrow bands with lower intensity at $2741-2698 \mathrm{~cm}^{-1}$ [29].These bands are shifted to $2929-2831 \mathrm{~cm}^{-1}, 2950-2875 \mathrm{~cm}^{-1}$ and $2947-2893 \mathrm{~cm}^{-1}$ in the cases of $1.0 \mathrm{wt} . \% \mathrm{PAC} / \mathrm{PEO}$, 1.0 wt.\% SM-PAC/PEO, and 2.0 wt.\% SM-AC/PEO, respectively. In relation to the $\mathrm{CH}_{2}$ stretching bands, which appear in the $1468-1242 \mathrm{~cm}^{-1}$ region, the most notable changes occur to the band at $1359 \mathrm{~cm}^{-1}$, which is shifted to $1353-1343$ and $1338 \mathrm{~cm}^{-1}$ in 1.0 wt.\% PAC/PEO, 1.0 wt.\% SM-PAC/PEO, and 2.0 wt.\% SM-PAC/PEO, respectively.

\subsection{Mechanical analysis}

The tensile strength, yield strength and percent strain values of virgin $\mathrm{PEO}, \mathrm{PAC} / \mathrm{PEO}$, and $\mathrm{SM}-\mathrm{PAC} / \mathrm{PEO}$ composites are given in Table I.

Table I reveals that the values (the tensile strength and the yield strength) obtained from the tensile testing for the PAC/PEO composite (1.0 wt.\%) have increased approximately two-fold, but its percent strain value decreased greatly, compared to the pure polymer. This
TABLE I

Results obtained from the tensile tests of pure PEO, $\mathrm{PAC} / \mathrm{PEO}$, and two SM-PAC/PEO composites.

\begin{tabular}{c|c|c|c}
\hline \hline Sample & $\begin{array}{c}\text { Tensile } \\
\text { strength } \\
{[\mathrm{MPa}]}\end{array}$ & $\begin{array}{c}\text { Yield } \\
\text { strength } \\
{[\mathrm{MPa}]}\end{array}$ & $\begin{array}{c}\% \\
\text { Strain }\end{array}$ \\
\hline Pure PEO & 0.781 & 0.547 & 54.64 \\
1.0 wt.\% PAC/PEO & 1.45 & 1.21 & 24.00 \\
1.0 wt.\% SM-AC/PEO & 1.38 & 0.97 & 82.74 \\
2.0 wt.\% SM-AC/PEO & 3.82 & 2.67 & 95.00
\end{tabular}

means that the unmodified powdered activated carbon dispersed into the polymer matrix caused it to be more brittle compared to pure polymer. However, the tensile and yield strengths of the composite containing modified powdered activated carbon, at the same ratio with the unmodified powdered activated carbon, have decreased relatively, but percent strain value has significantly increased. Although percent strain is not accepted as an absolute measure for ductility, the increase in its value can be interpreted as though the composite with the capacity to absorb much more deformation energy after the addition of modified powdered activated carbon has gained a higher elastic character. This indicates that the prepared composite with the ratio of $2.0 \mathrm{wt} . \%$ of the modified powder activated carbon has been significantly reinforced, showing remarkable improvements in the ductility of polymer.

\subsection{Thermal analysis}

The results obtained by the DSC analysis of pure PEO, $\mathrm{PAC} / \mathrm{PEO}$, and two SM-PAC/PEO composites are given in Table II. This table shows that values of the crystallinity percentage for PAC/PEO composite (1.0 wt.\%) and two other composites approximately have increased two-fold but their $T_{m}$ values have decreased slightly, compared to those of the pure polymer. This indicates that the modified and unmodified powdered activated carbon has caused a conformational change which leads to a more ordered rearrangement of the polymer chains.

TABLE II

Results from DSC analysis of pure PEO, PAC/PEO, and two SM-PAC/PEO composites.

\begin{tabular}{c|c|c|c}
\hline \hline Sample & $\begin{array}{c}T_{m} \\
{\left[{ }^{\circ} \mathrm{C}\right]}\end{array}$ & $\begin{array}{c}\Delta H_{m} \\
{\left[\mathrm{~J} \mathrm{~g}^{-1}\right]}\end{array}$ & $\begin{array}{c}\text { Crystallinity } \\
{[\%]}\end{array}$ \\
\hline Pure PEO & 66.72 & 101.65 & 43.87 \\
1.0 wt.\% PAC/PEO & 69.60 & 176.00 & 76.00 \\
1.0 wt.\% SM-AC/PEO & 67.70 & 164.00 & 71.00 \\
2.0 wt.\% SM-AC/PEO & 68.60 & 169.00 & 73.00
\end{tabular}

\section{Conclusions}

The main results of this study are summarized below:

- SM-PAC/PEO composites were successfully prepared and characterized. 
- The SEM images of the surfactant-modified powdered activated carbon (SM-PAC)/PEO composites prepared in the two different SM-PAC contents show that there is an intensive interaction between the $\mathrm{CTA}^{+}$ions of modified activated carbon surface and the polymer chains.

- The results of the tensile test indicate that very significant improvements in the mechanical properties of composites emerged, compared to those of the pure polymer. This indicates that the presence of activated carbon particles positively affects the conformation of the polymer chains.

- DSC analysis reveals that the modified and unmodified powdered activated carbon causes a conformational change which leads to the more ordered rearrangement of polymer chains.

\section{References}

[1] C. Akmil Başar, A. Karagunduz, B. Keskinler, A. Cakici, Appl. Surf. Sci. 218, 170 (2003).

[2] H-J. Hong, H. Kim, Y-J. Lee, J-W. Yang, J. Hazard. Mater. 170, 1242 (2009).

[3] C. Yan, L. Zou, R. Short, Desalination 333, 101 (2014).

[4] M. Anson, J. Marchese, E. Garis, N. Ochoa, C. Pagliero, J. Membrane Sci. 243, 19 (2004).

[5] L. Ballinas, C. Torras, V. Fierro, R. Garcia-Valls, J. Phys. Chem. Solids 65, 633 (2004).

[6] A. Oh, J. Yun, H.-I. Kim, J. Polym. Res. 18, 2441 (2011).

[7] D.R. Simpson, Water Res. 42, 2839 (2008).

[8] L.P. Ma, P. Ning, Y.Y. Zhang, X.Q. Wang, Chem. Eng. J. 137, 471 (2008).

[9] D. Mehandjiev, M. Khristova, E. Bekyarova, Carbon 34, 757 (1996).

[10] H. Fukuyama, S. Terai, Catal. Today 130, 382 (2008).
[11] L.S. Chan, W.H. Cheung, S.J. Allen, G. McKay, Sep. Purif. Technol. 67, 166 (2009).

[12] H. Naono, M. Hakuman, M. Shimoda, K. Nakai, S. Kondo, J. Colloid Interface Sci. 182, 230 (1996).

[13] J. Hu, Q.M. Gao, Y.H. Wu, S.Q. Song, Int. J. Hydrogen Energy 32, 1943 (2007).

[14] L. Feng, J. Zheng, H. Yang, Y. Guo, W. Li, X. Li, Solar Energ. Mat. Sol. C. 95, 644 (2011).

[15] K. Baek, B.-K. Kim, H.-J. Cho, J.-W. Yang, J. Hazard. Mater. 99, 303 (2003).

[16] H.-D. Choi, M.-C. Shin, D.-H. Kim, C.-S. Jeon, K. Baek, Desalination 223, 290 (2008).

[17] C.A. Basar, C. Aydiner, S. Kara, B. Keskinler, Sep. Purif. Technol. 48, 270 (2006).

[18] K. Baek, B.-K. Kim, J.-W. Yang, Desalination 156, 137 (2003).

[19] K. Baek, J.-W. Yang, Desalination 167, 101 (2004).

[20] H.-D. Choi, J.-M. Cho, K. Baek, J.-S. Yang, J.-Y. Lee, J. Hazard. Mater. 161, 1565 (2009).

[21] J. Kahovec, R.B. Fox, K. Hatada, Pure Appl. Chem. 74, 1921 (2002).

[22] K. Pielichowski, K. Flejtuch, J. Anal. Appl. Pyrol. 73, 131 (2005).

[23] M.M. Crowley, F. Zhang, J.J. Koleng, J.W. McGinity, Biomaterials 23, 4241 (2002).

[24] N.S. Vrandecic, M. Erceg, M. Jakic, I. Klaric, Thermochim. Acta 498, 71 (2010).

[25] M.M.E. Jacob, E. Hackett, E.P. Giannelis, J. Mater. Chem. 13, 1 (2003).

[26] Z. Shen, G.P. Simon, Y-B. Cheng, Polymer 43, 4251 (2002).

[27] W. Loyens, P. Jannasch, F.H.J. Maurer, Polymer 46 915 (2005).

[28] P. Meneghetti, S. Qutubuddin, J. Colloid Interface Sci. 288, 387 (2005).

[29] P. Aranda, E. Ruiz-Hitzky, Acta Polym. 45, 59 (1994). 